\title{
ASSOCIATION BETWEEN TINNITUS AND MENTAL HEALTH AMONG KOREAN ADOLESCENTS: THE KOREA NATIONAL HEALTH AND NUTRITION EXAMINATION SURVEY
}

\author{
Ji-Su Kim \\ Department of Nursing, Chung-Ang University, Seoul, Republic of Korea
}

\section{SUMMARY}

Objectives: The pathophysiology of tinnitus is not clearly understood, but its prevalence is relatively high. Persistent symptoms of tinnitus are linked to various mental health conditions, and in particular, tinnitus prevalence is high in Korean adolescents. Accordingly, this study aimed to reveal the associations between tinnitus and mental health in Korean adolescents using a nationally representative sample.

Methods: Original data from the Korea National Health and Nutrition Examination Survey were analyzed by logistic regression analysis. The sample consisted of 1,587 adolescents aged 12-18. We examined demographic variables; tinnitus measured by health examination; and stress, depression, and suicidal ideation measured by health interview.

Results: The prevalence of tinnitus was $18.0 \%$. Tinnitus severity was directly related to level of stress $(p=0.023)$, depression $(p<0.001)$, and suicidal ideation $(p<0.001)$. In comparison with Korean adolescents without tinnitus, the odds ratio (OR) of having depression and suicidal ideation was 2.27 (95\% Cl: $1.47-3.50)$ and 1.96 (95\% Cl: 1.35-2.84), respectively, in univariable analysis. The OR of having depression and suicidal ideation in multivariate analysis was $2.00(95 \% \mathrm{Cl}: 1.28-3.13)$ and $1.73(95 \% \mathrm{Cl}: 1.13-2.62)$, respectively.

Conclusions: Tinnitus prevalence in Korean adolescents is relatively high, and thus, intervention may be necessary to prevent or reduce depression and suicidal ideation to ensure that other areas of life are not negatively affected. Further, individuals with tinnitus may be in need of intervention to prevent or reduce depression and suicidal ideation.

Key words: tinnitus, mental health, adolescent, Korea, depression, suicide ideation

Address for correspondence: Ji-su Kim, Department of Nursing, Chung-Ang University, 84 Heukseok-Ro, Dongjack-Gu, Seoul 156-756, Republic of Korea. E-mail: jisu80@cau.ac.kr

https://doi.org/10.21101/cejph.a4514

\section{INTRODUCTION}

Tinnitus refers to the perception of a sound in the absence of any external stimuli. Subjective tinnitus is the most frequent type of tinnitus, and patients often describe it as a ringing or buzzing sound in one or both ears $(1,2)$. The pathophysiology of tinnitus remains poorly understood, but its prevalence is known to be relatively high across all ages (1). The reported prevalence of tinnitus in adults varies from $15 \%$ to $35 \%$, and it increases with age (3). Further, although it is challenging to measure the prevalence of tinnitus in children, it is reported to be approximately equivalent with the prevalence in adults. Approximately $37 \%$ of children report tinnitus sensations, and $17 \%$ report tinnitus annoyance $(4$, 5). In particular, as adolescents expose themselves to loud music and excessive noise during social and music events (6-9), the risk of tinnitus is likely to be higher as compared to other age groups. However, tinnitus remains underappreciated in this population because of underreporting $(1,10)$.

The National Health and Nutrition Examination Survey (NHANES) revealed that the prevalence of tinnitus in adolescents in the US is $5.7-9.5 \%$ (10). On the other hand, the Korea
NHANES (KNHANES) reported that the prevalence of tinnitus in adolescents is $20 \%$ or higher, even though most have normal hearing. Further, the prevalence of tinnitus in adolescents was higher than it was among adults aged 30-40 years (3).

If subjective perception of a sound caused by tinnitus continues, it may interfere with activities of daily living (11). Previous studies have reported that tinnitus may negatively influence concentration, sleep, completion of tasks, and social interaction $(12,13)$, and consequently, some individuals with tinnitus may be more likely to be depressed $(5,11,14-18)$. Further, tinnitus symptoms have been linked to various mental health (19-24).

Despite the relatively high prevalence of tinnitus among youth, until now, studies on tinnitus in adolescents have been limited to the perspective of a single institution or a small sample size, and the authenticity of these numbers has never been investigated by means of a population-based database. In Korea, studies on tinnitus have been limited to its association with hypacusis and noise, as well as chronic disease in adults. Studies on tinnitus among youth have been limited to investigating its prevalence and risk factors (1), and only a few studies have focused on the association between tinnitus and mental health in youth. 
In Korea, nearly 1 in 5 adolescents reports having tinnitus, and the perceived stress in youth is higher than it is in adults. In addition, about 1 in 20 adolescents has attempted suicide at least once, as the youth population suffers from fierce academic competition (25). Therefore, Korean youth are more likely to have unfavourable mental health conditions such as stress, depression, and suicidal ideation as compared to other age groups, leading to a relatively high prevalence of tinnitus. Thus, it is important to investigate the association between tinnitus and mental health. This study aimed to investigate the association between tinnitus and mental health in Korean adolescents with a nationally representative sample. Ultimately, the findings might help to reduce tinnitus-related problems and improve the mental health of Korean youth.

\section{MATERIALS AND METHODS}

\section{Study Population}

We used the first (2010) through third (2012) year data of the Korea National Health and Nutrition Examination Survey V (KNHANES V) conducted by the Korea Centres for Disease Control and Prevention (KCDC). We requested to use the data by filling in the data use plan and written pledge on the homepage of the KNHANES V, and then received KDCD approval for data use. The purpose of the KNHANES investigation is to produce statistical data on Korean people's health status, awareness, and behaviours with full representativeness and reliability at the national, provincial, and city levels. The statistics have been provided to the World Health Organization and Organization for Economic Co-operation and Development.

In the present study, the 3-year data of the KNHANES V (2010-2012) constituted an independent probability sample representing the whole nation, and the rolling survey sampling method was used so that samples with similar year-specific characteristics were selected. For sampling, cities and provinces were primarily aggregated, and then secondarily aggregated according to gender, population ratio, and so forth. From each of the extracted sample enumeration districts, 20 final households subject to this research investigation were extracted. Of the final samples, people aged at least 1 year were the subjects. We analyzed data of 1,587 adolescents, excluding 242 who did not respond to the relevant surveys.

\section{Measures}

Tinnitus. The presence of tinnitus was defined as responding "yes" to the question: "In the past 12 months, have you been bothered by buzzing in your ears?" The severity of tinnitus was graded as "no problem", "bothering", and "having trouble sleeping" to the question: "How much of a problem is this ringing in your ears?"

Mental health. Mental health-related variables included recognition of stress, experience of depression, and experience of suicidal ideation. Stress was categorized by grouping participants' answers of "I feel stressed very much", "I feel stressed a lot", and "I feel stressed a little" into "Yes" and "I hardly feel stressed" into "No". Depressive symptoms were analyzed by asking "Have you felt sad or depressed for at least 2 consecutive weeks within the last year to the extent of disturbing your daily life?" Participants answered "Yes" or "No" to this question. Suicidal thoughts were assessed by the question "Have you ever thought of committing suicide in the last year?" Again, participants responded with "Yes" or "No".

Other variables. Demographic and health-related characteristics used were gender, age, living place, economic status, overweightness, using headphones in a noisy place, tympanic membrane status, current smoker, current drinker, regular exercise, and sleep time. Age was divided into middle school students (12-14) and high school students (15-18). Living area was categorized as urban or rural. Household economic status was analyzed by equivalent income (i.e., average monthly household income divided by the square root of the number of household members) for the bottom $25 \%$, which adjusts household income by the number of household members. Body mass index (BMI), one of growth indexes of Korean children and adolescents, was categorized into two groups: below the 85 th percentile and equal to or above 85 th percentile. Using headphones in a noisy place was also categorized into two groups based on "Yes" or "No" responses to the question, "Have you ever put on headphones or earphones in a loud place such as a bus or subway and listened to loud music?"

Tympanic membrane status was also categorized into two groups: tympanic membrane in an unfavourable condition (e.g., perforation, inflammation) and tympanic membrane with no unfavourable conditions. Smoking status was determined by the subjects' reporting of whether they had never smoked, had smoked in the past, or were current smokers. As for drinking status, subjects who indicated "Drinking more than once a month" were considered to be current drinkers. Regular exercise was defined as strenuous physical activity performed for at least $20 \mathrm{~min}$ at a time at least three times a week. Sleep time was defined as the average daily sleep hours indicated by subjects.

\section{Analyses}

Statistical analyses were conducted using SAS survey procedures (version 9.3; SAS Institute, Cary, N.C., USA), in a manner that reflected sampling weights and provided nationally representative estimates according to KCDC guidelines. Logistic regression analyses were used to determine the association between tinnitus and mental health among adolescents. Odds ratios (ORs) and confidence intervals (CIs) were estimated after adjusting for age, sex, BMI, economic status, using headphones in a noisy place, and sleep time. A p-value of $<0.05$ was considered statistically significant.

\section{RESULTS}

The prevalence and severity of tinnitus among adolescents are shown in Table 1. The prevalence of tinnitus was shown to be $18.0 \%$, and $18.1 \%$ of the youth population reporting tinnitus answered "bothering", and $2.7 \%$ responded "having trouble sleeping" regarding the extent to which tinnitus is a problem.

Differences in tinnitus according to demographic characteristics among adolescents are shown in Table 2. First, the prevalence of tinnitus was $43.0 \%$ for males and $57.0 \%$ for females 
Table 1. Prevalence and severity of tinnitus among adolescents $(N=1,587)$

\begin{tabular}{|l|c|}
\hline Variables & $\%($ SE) \\
\hline Prevalence of tinnitus & $18.0(1.4)$ \\
\hline Severity of tinnitus \\
\hline No problem & $79.1(1.4)$ \\
\hline Bothering & $18.1(1.4)$ \\
\hline Having trouble sleeping & $2.7(0.5)$ \\
\hline
\end{tabular}

$\left(\chi^{2}=11.888, \mathrm{p}<0.001\right)$. Prevalence was $41.5 \%$ among 12 - to 14-year-old subjects, and prevalence was higher among high school students $\left(\chi^{2}=7.445, p=0.019\right)$. The prevalence of tinnitus was $52.6 \%$ for subjects who used headphones in a noisy place, and $47.4 \%$ for subjects who did not $\left(\chi^{2}=5.999, p<0.001\right)$. Sleep time was 7.10 hours for subjects reporting tinnitus and 6.82 hours for those not reporting tinnitus $(\mathrm{t}=123.16, \mathrm{p}<0.001)$. Moreover,
$19.2 \%$ of adolescents with an experience of stress reported having tinnitus $\left(\chi^{2}=14.626, p<0.001\right)$, and $21.5 \%$ of adolescents with an experience of suicidal ideation reported having tinnitus $\left(\chi^{2}=13.421, p<0.001\right)$. On the other hand, tinnitus was more prevalent in subjects from urban areas, those who were current smokers, current drinkers, those with an economic status in the first quartile, and those who recognized stress. However, these effects were non-significant.

Mental health according to severity of tinnitus among adolescents is illustrated in Figure 1. Among adolescents with an experience of stress, $24.5 \%$ reported "no problem", $26.7 \%$ "bothering", and $46.0 \%$ "having trouble sleeping" regarding the extent to which tinnitus is a problem. In other words, the severity of tinnitus is directly associated with the level of perceived stress $(p=0.022)$. In addition, among adolescents with an experience of depression, $8.7 \%$ reported "no problem", $16.3 \%$ "bothering", and $28.4 \%$ "having trouble sleeping", representing a significant difference from those without experience of depression $(p<0.001)$. Among youth with an experience of suicidal ideation, $12.2 \%$

Table 2. Tinnitus according to demographic characteristics $(N=1,587)$

\begin{tabular}{|c|c|c|c|c|c|c|}
\hline \multirow{2}{*}{\multicolumn{2}{|c|}{ Variables }} & \multirow{3}{*}{$\begin{array}{c}n(\%) \\
848(53.6) \\
\end{array}$} & \multicolumn{2}{|c|}{ Tinnitus } & \multirow{4}{*}{$\begin{array}{l}\mathrm{X}^{2} \text { or } \mathrm{t} \\
11.888\end{array}$} & \multirow{4}{*}{$\begin{array}{c}\mathrm{p} \\
<0.001\end{array}$} \\
\hline & & & \multirow{2}{*}{$\begin{array}{c}\text { No } \\
\% \text { (SE) or } \mathrm{M} \pm \mathrm{SE}\end{array}$} & \multirow{2}{*}{$\begin{array}{c}\text { Yes } \\
\% \text { (SE) or M } \pm \text { SE }\end{array}$} & & \\
\hline \multirow{2}{*}{ Gender } & Male & & & & & \\
\hline & Female & 739 (46.4) & $43.6(1.7)$ & $57.0(3.2)$ & & \\
\hline \multirow{2}{*}{ Age (years) } & $12-14$ & 883 (48.1) & $49.9(1.6)$ & $41.5(3.2)$ & \multirow{2}{*}{7.445} & \multirow{2}{*}{0.019} \\
\hline & $15-18$ & $704(51.9)$ & $50.1(1.6)$ & $58.5(3.2)$ & & \\
\hline \multirow{2}{*}{ Living place } & Rural & $233(17.9)$ & $16.9(2.5)$ & $21.5(4.0)$ & \multirow{2}{*}{2.050} & \multirow{2}{*}{0.152} \\
\hline & Urban & $1,354(82.1)$ & $83.1(2.5)$ & $78.5(4.0)$ & & \\
\hline \multirow{2}{*}{ Economic status } & $1 Q$ & $1,356(83.8)$ & $84.0(1.5)$ & $82.6(3.0)$ & \multirow{2}{*}{0.258} & \multirow{2}{*}{0.611} \\
\hline & $\geq 2 Q$ & $204(16.2)$ & $16.0(1.5)$ & $17.4(3.0)$ & & \\
\hline \multirow{2}{*}{ BMI $\left(\mathrm{kg} / \mathrm{m}^{2}\right)$} & $<85$ percentile & $1,303(81.9)$ & $81.8(1.3)$ & $81.9(2.6)$ & \multirow{2}{*}{0.001} & \multirow{2}{*}{0.977} \\
\hline & $\geq 85$ percentile & $284(18.1)$ & $18.2(1.3)$ & $18.1(2.6)$ & & \\
\hline \multirow{2}{*}{$\begin{array}{l}\text { Using headphones } \\
\text { in a noisy place }\end{array}$} & None & $604(40.4)$ & $38.6(2.0)$ & $47.4(3.4)$ & \multirow{2}{*}{5.999} & \multirow{2}{*}{0.014} \\
\hline & Have & $979(59.6)$ & $61.4(2.0)$ & $52.6(3.4)$ & & \\
\hline \multirow{2}{*}{$\begin{array}{l}\text { Tympanic } \\
\text { membrane }\end{array}$} & Normal & $1,570(99.2)$ & $99.1(0.3)$ & $99.5(0.3)$ & \multirow{2}{*}{0.666} & \multirow{2}{*}{0.331} \\
\hline & Abnormal & $15(0.8)$ & $0.9(0.3)$ & $0.5(0.3)$ & & \\
\hline \multirow{2}{*}{ Current smoker } & No & 1,307 (78.1) & $78.9(1.6)$ & $75.0(2.8)$ & \multirow{2}{*}{1.377} & \multirow{2}{*}{0.240} \\
\hline & Yes & $280(21.9)$ & $21.1(1.6)$ & $25.0(2.8)$ & & \\
\hline \multirow{2}{*}{ Current drinker } & No & $1,154(66.7)$ & $68.2(1.8)$ & $61.1(3.6)$ & \multirow{2}{*}{3.616} & 0057 \\
\hline & Yes & $432(33.3)$ & $31.8(1.8)$ & $38.9(3.6)$ & & 0.001 \\
\hline Reoular oyorrico & No & 1,088 (68.6) & $68.0(1.5)$ & $71.0(3.3)$ & 0642 & 0.422 \\
\hline 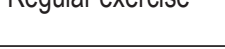 & Yes & 496 (31.4) & $32.0(1.5)$ & $29.0(3.3)$ & & $0.4 \angle C$ \\
\hline Sleep time (hours) & & & $7.10 \pm 0.05$ & $6.82 \pm 0.08$ & 7.09 & $<0.001$ \\
\hline Recognition of & No & $1,189(74.6)$ & $75.5(1.6)$ & $70.8(2.8)$ & 2315 & 128 \\
\hline stress & Yes & $398(25.4)$ & $24.5(1.6)$ & $29.2(2.8)$ & 2.010 & 0.120 \\
\hline Experience of & No & $1,436(89.3)$ & $91.3(1.0)$ & $82.1(2.8)$ & 11606 & $<0001$ \\
\hline depression & Yes & $151(10.7)$ & $8.7(1.0)$ & $17.9(2.8)$ & $17.0<0$ & .0 .001 \\
\hline Experience of & No & 1,363 (85.8) & $87.8(1.1)$ & $78.5(2.6)$ & 13.421 & $<0.001$ \\
\hline suicidal ideation & Yes & $224(14.2)$ & $12.2(1.1)$ & $21.5(2.6)$ & 10.421 & $<.001$ \\
\hline
\end{tabular}




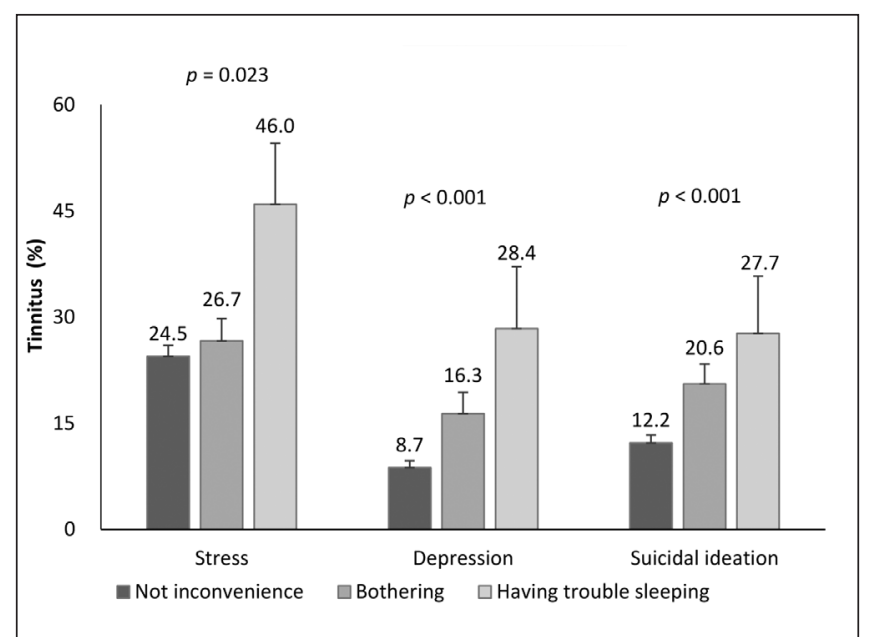

Fig. 1. Mental health according to tinnitus severity.

reported "no problem", 20.6\% "bothering", and 27.7\% "having trouble sleeping", representing significant differences from those without experience of suicide ideation $(p<0.001)$. In the youth population, tinnitus severity was shown to be directly associated with negative mental health outcomes.

The association between tinnitus and mental health among adolescents is shown in Table 3. In univariable analysis, the comparison of subjects reporting having tinnitus and those without tinnitus showed that the OR of having depression was 2.27 (95\% CI: 1.47-3.50), and the OR of having suicidal ideation was 1.96 (95\% CI: 1.35-2.84) for subjects with tinnitus. Further, in multivariable analysis (adjusted for age, sex, BMI, economic status, using headphones in a noisy place, and sleep time), the comparison of subjects reporting having tinnitus and those without tinnitus showed that the OR of having depression was $2.00(95 \%$ CI: 1.28-3.13), and the OR of having suicidal ideation was 1.73 (95\% CI: 1.13-2.62) for subjects with tinnitus.

\section{DISCUSSION}

Among Korean adolescents, those who perceived the severity of tinnitus to be a problem and those who were bothered with tinnitus were more likely to have depression and suicidal ideation. Although the main focus of this study was the association between tinnitus and mental health among Korean adolescents, preliminary analyses examined the prevalence and factors affecting tinnitus.

The prevalence of tinnitus in the youth population of South Korea has reached $18.0 \%$, and among adolescents with tinnitus, tinnitus was moderate or severe in $20 \%$. This result is consistent with a small-scale survey in Belgium that found the prevalence of temporary tinnitus to be $74.9 \%$ and that of permanent tinnitus to be $18.3 \%$ among high school students. However, these prevalence values are higher than those found in a national sample in the US, which reported the prevalence of tinnitus to be $5.7 \%$ in 12 - to 15 -year olds and $9.5 \%$ in 16 - to 19 -year olds (10). Although the prevalence of tinnitus in the paediatric population has been investigated in a few epidemiological studies (10), it is hard to accurately analyze the severity and/or prevalence of tinnitus and make a comparative analysis because tinnitus is a subjective symptom. Accordingly, the prevalence of tinnitus in the youth population could be underestimated because adolescents might have more difficulties expressing their symptoms than adults do (1). Thus, the prevalence of tinnitus among youth in Korea appears to be relatively high. It is necessary to develop appropriate tools for assessment of tinnitus to evaluate both physical and psychological health status of the youth population.

This study revealed that the prevalence of tinnitus is the highest in adolescents with the following characteristics: aged 15-18 years, female gender, history of using headphones in a noisy place, and short sleeping hours. These findings are consistent with the nationally representative US study (10), which claimed that risk factors of tinnitus in US adolescents are female gender and recreational noise exposure history. It should be noted that each study claims a different factor attributable to the higher prevalence of tinnitus in females. The US study (10) emphasizes that there is an association between female gender and low income, and Korean and Japan studies $(3,14,27)$ claim that the heightened prevalence in females can be attributed to a more stressful cultural situation for women in Asia, which demands obedience and a greater family role. Alternatively, women may be more likely to report any tinnitus, even if it is just annoying. Still, all these previous studies have agreed that the prevalence of tinnitus is high in females in all ages including adolescents $(1,10)$, adults, and seniors $(3,14,27-29)$, and it is necessary to investigate the role of gender by culture and age.

Hearing loss is a known major risk factor of tinnitus $(5,14,22$, 28-30), and causes of hearing loss might vary by age. The risk of permanent hearing loss in adolescents has increased dramatically with the rise in exposure to high noise levels, as adolescents are likely to use smartphones and MP3 players, and consequently, music-induced hearing loss may evolve into a significant social and public health problem $(6,7)$. Therefore, changes in these risky sound exposure behaviours are needed for preventing tinnitusrelated hearing loss in adolescents (9). Insomnia is known to be the second-most frequent symptom, following hearing loss, in patients with tinnitus (31). However, the pathophysiological

Table 3. Association between tinnitus and mental health

\begin{tabular}{|l|l|c|c|}
\hline \multicolumn{2}{|c|}{} & Depression & Suicidal ideation \\
\hline \multirow{2}{*}{ Model 1 } & Tinnitus (No) & 1 & 1 \\
\cline { 2 - 4 } & Tinnitus (Yes) & $2.27(1.47-3.50)$ & $1.96(1.35-2.84)$ \\
\hline \multirow{2}{*}{ Model 2 } & Tinnitus (No) & 1 & 1 \\
\cline { 2 - 4 } & Tinnitus (Yes) & $2.00(1.28-3.13)$ & $1.73(1.13-2.62)$ \\
\hline
\end{tabular}

Model 1: univariate; Model 2: adjusted for age, sex, BMI, economic status, using headphones in a noisy place, and sleep time. 
mechanism of the association between tinnitus and sleep disorders has not been revealed, and there is only a limited amount of research on this issue (13). This study showed that sleeping hours were significantly shorter in adolescents with tinnitus. In Europe, the percentage of adolescents sleeping 8 hours a night or more during weekdays is $62 \%$ (32), whereas it is only $15.5 \%$ in Korea, demonstrating the lack of sleep among Korean youth (33). Adolescence is a period in which circadian rhythms go through a dramatic change, and many adolescents are likely to suffer from sleep deprivation as well as sleeping disorders because they start to sleep at later hours but the time at which they go to school is fixed $(33,34)$. It is difficult to determine the causal relationship between tinnitus and sleep time with the limited amount of data obtained from this study, but because this study has verified the association of tinnitus with depression and suicidal ideation, it is necessary to create an environment that facilitates adequate sleep among adolescents in order to reduce negative outcomes of tinnitus in youth.

According to this study, adolescents with depression and suicidal ideation are likely to report having tinnitus, and the perceived levels of stress, depression, and suicidal ideation are higher among those with more severe tinnitus. Further, we demonstrated an association of tinnitus with depression and suicidal ideation, but not stress. This result can only be compared with previous studies among adults and seniors, as no previous studies have targeted youth. Adolescents with annoying tinnitus had higher odds of having stress and depression than did adolescents with any other types of tinnitus $(3,27)$. Further, depression scores increase with the severity of tinnitus (11), and depression can be used to estimate tinnitus severity (18). In this study, among various mental health factors, only the recognition of stress did not show a statistically significant outcome, which might be because the definition of stress varies by study, and stress is the weakest symptom of mental problems. However, this issue still requires additional research to verify the association of tinnitus with stress in youth.

A number of previous studies $(5,11,14-18)$ reported the association of tinnitus with depression, but a recent large populationbased study showed a much weaker correlation between depression and tinnitus severity (21). This might be because symptom overlap between tinnitus and depression works as a confounder, and the pathophysiology of tinnitus and depression argue against comorbidity by chance and against depression as a pure reaction to tinnitus. Instead, there may be a complex interplay between tinnitus and depression (17). Further, a few previous studies have argued that there is no clear evidence of the association of tinnitus and suicide (35), while others have claimed that the association of tinnitus and suicide indicates the role of tinnitus as a "precipitant" (36). Despite such contradictory arguments, the significant association of tinnitus with depression and suicidal ideation was shown when the confounders of depression and suicidal ideation were accounted for in this study, and this finding necessitates a replication study on tinnitus in the youth population.

Taken together, these findings suggest that individuals with tinnitus may require intervention to prevent or reduce depression and suicidal ideation so as to ensure that other areas of their lives are not negatively influenced. Potential evidence-based strategies among adolescents should seek to care for tinnitus, and ultimately depression and suicidal ideation, including psychological treatment (11).
This study has a few limitations. First, tinnitus is a self-reported and subjective symptom, and so it might not be appropriate to interpret the findings as absolute. Further, as this was a crosssectional study, it is not possible to verify a causative relationship between tinnitus and mental health. Nevertheless, this study is the first study to verify the association between tinnitus and mental health in adolescents using nationally representative data, and the findings represent the first evidence to support the importance of the assessment and management of tinnitus in the development of interventions aimed at preventing or reducing depression and suicidal ideation in adolescents.

\section{CONCLUSIONS}

We found that $18 \%$ of the community-dwelling Korean adolescents aged 12-18 years had tinnitus. Depression and suicidal ideation were associated with tinnitus in this population. Thus, intervention may be necessary to prevent or reduce depression and suicidal ideation to ensure that other areas of life are not negatively affected. Further, individuals with tinnitus may be in need of intervention to prevent or reduce depression and suicidal ideation. A prospective study or randomized trials are also required to clarify the causality.

\section{Acknowledgements}

The data for this study were provided by the Korea Centres for Disease Control and Prevention.

\section{Conflict of Interests}

None declared

\section{REFERENCES}

1. Kim CY, Jeong SW, Seo JY, Lee YJ, Park SC, Choi HS. The Prevalence and risk factors of tinnitus in Korean adolescents. Korean J Otorhinolaryngol Head Neck Surg. 2015;58(1):12-8

2. Shetye A, Kennedy V. Tinnitus in children: an uncommon symptom? Arch Dis Child. 2010;95(8):645-8.

3. Park RJ, Moon JD. Prevalence and risk factors of tinnitus: the Korean National Health and Nutrition Examination Survey 2010-2011, a crosssectional study. Clin Otolaryngol. 2014;39(2):89-94.

4. Coelho CB, Sanchez TG, Tyler RS. Tinnitus in children and associated risk factors. Prog Brain Res. 2007;166:179-91.

5. Baguley D, McFerran D, Hall D. Tinnitus. Lancet. 2013;382(9904):16007.

6. Bohlin MC, Erlandsson SI. Risk behaviour and noise exposure among adolescents. Noise Health. 2007;9(36):55-63.

7. Vogel I, Brug J, Hosli EJ, van der Ploeg CP, Raat H. MP3 players and hearing loss: adolescents' perceptions of loud music and hearing conservation. J Pediatr. 2008;152(3):400-4.

8. Vogel I, Brug J, van der Ploeg CP, Raat H. Young people's exposure to loud music: a summary of the literature. Am J Prev Med. 2007;33(2):124-33.

9. Rossa KR, Smith SS, Allan AC, Sullivan KA. The effects of sleep restriction on executive inhibitory control and affect in young adults. J Adolesc Health. 2014;55(2):287-92.

10. Mahboubi H, Oliaei S, Kiumehr S, Dwabe S, Djalilian HR. The prevalence and characteristics of tinnitus in the youth population of the United States. Laryngoscope. 2013;123(8):2001-8.

11. Loprinzi PD, Maskalick S, Brown K, Gilham B. Association between depression and tinnitus in a nationally representative sample of US older adults. Aging Ment Health. 2013;17(6):714-7.

12. Dobie RA. Depression and tinnitus. Otolaryngol Clin North Am. 2003;36(2):383-8. 
13. Kim SI, Kim TH, Byun YS, Chung JH, Kim SJ, Park MS, et al. Association between insomnia and depression in tinnitus patients. Korean $\mathrm{J}$ Otorhinolaryngol Head Neck Surg. 2015;58:313-7.

14. Michikawa T, Nishiwaki Y, Kikuchi Y, Saito H, Mizutari K, Okamoto M, et al. Prevalence and factors associated with tinnitus: a community-based study of Japanese elders. J Epidemiol. 2010;20(4):271-6.

15. Geocze L, Mucci S, Abranches DC, Marco MAD, Penido NDO. Systematic review on the evidences of an association between tinnitus and depression. Braz J Otorhinolaryngol. 2013;79(1):106-11.

16. Falkenberg ES, Wie OB. Anxiety and depression in tinnitus patients: 5-year follow-up assessment after completion of habituation therapy. Int J Otolaryngol. 2012;2012:375460. doi:10.1155/2012/375460.

17. Langguth B, Landgrebe M, Kleinjung T, Sand GP, Hajak G. Tinnitus and depression. World J Biol Psychiatry. 2011;12(7):489-500.

18. Ooms E, Meganck R, Vanheule S, Vinck B, Watelet JB, Dhooge I. Tinnitus severity and the relation to depressive symptoms: a critical study. Otolaryngol Head Neck Surg. 2011;145(2):276-81.

19. Zöger S, Svedlund J, Holgers KM. Relationship between tinnitus severity and psychiatric disorders. Psychosomatics. 2006;47(4):282-8.

20. Pinto P, Marcelos C, Mezzasalma M, Osterne FJV, de Melo Tavares de Lima MA, Nardi AE. Tinnitus and its association with psychiatric disorders: systematic review. J Laryngol Otol. 2014;128(8):660-4.

21. Krog NH, Engdahl B, Tambs K. The association between tinnitus and mental health in a general population sample: results from the HUNT Study. J Psychosom Res. 2010;69(3):289-98.

22. Meinke DK, Martin WH, Griest SE, Howart L, Sobel JL, Scarlotta T. Dangerous Decibels ${ }^{\circledR}$ I: Noise induced hearing loss and tinnitus prevention in children. Noise exposures, epidemiology, detection, interventions and resources. In: Griefahn B, editor. Noise as a public health problem. ICBEN 2008: the 9th Congress of the International Commission on the Biological Effects of Noise: proceedings; 2008 Jul 21-25; Mashantucket, Connecticut, USA. Dortmund: IfADo; 2008. p. 62-70.

23. Brunnberg E, Lindén-Boström M, Berglund M. Tinnitus and hearing loss in 15-16-year-old students: mental health symptoms, substance use, and exposure in school. Int J Audiol. 2008;47(11):688-94.

24. Figueiredo RR, Rates MA, Azevedo AA, Oliveira PMD, Navarro P. Correlation analysis of hearing thresholds, validated questionnaires and psychoacoustic measurements in tinnitus patients. Braz J Otorhinolaryngol. 2010;76(4):522-6.
25. Park EN. The influencing factors on suicide attempt among adolescents in South Korea. J Korean Acad Nurs. 2008;38:465-73.

26. Gilles A, Van Hal G, De Ridder D, Wouters K, Van de Heyning P. Epidemiology of noise-induced tinnitus and the attitudes and beliefs towards noise and hearing protection in adolescents. PLoS One. 2013;8(7):e70297. doi: 10.1371/journal.pone.0070297.

27. Park KH, Lee SH, Koo JW, Park HY, Lee KY, Choi YS, et al. Prevalence and associated factors of Tinnitus: data from the Korean National Health and Nutrition Examination Survey 2009-2011. J Epidemiol. 2014;24(5):417-26.

28. Sindhusake D, Golding M, Wigney D, Newall P, Jakobsen K, Mitchell P. Factors predicting severity of tinnitus: a population-based assessment. J Am Acad Audiol. 2004;15(4):269-80.

29. Shargorodsky J, Curhan GC, Farwell WR. Prevalence and characteristics of tinnitus among US adults. Am J Med. 2010;123(8):711-8.

30. Gilles A, De Ridder D, Van Hal G, Wouters K, Punte AK, Van de Heyning P. Prevalence of leisure noise-induced tinnitus and the attitude toward noise in university students. Otol Neurotol. 2012;33(6):899-906.

31. Asplund R. Sleepiness and sleep in elderly persons with tinnitus. Arch Gerontol Geriatr. 2003;37(2):139-45.

32. Garaulet M, Ortega F, Ruiz J, Rey-Lopez, J P, Beghin L, Manios Y, et al. Short sleep duration is associated with increased obesity markers in European adolescents: effect of physical activity and dietary habits. The HELENA study. Int J Obes (Lond). 2011;35(10):1308-17.

33. Park H. Effect of sleep duration on suicidal ideation in Korean Adolescents. J Korean Soc Sch Health. 2015;28:1-9.

34. Fitzgerald CT, Messias E, Buysse DJ. Teen sleep and suicidality: results from the youth risk behavior surveys of 2007 and 2009. J Clin Sleep Med. 2011;7(4):351-6.

35. Jacobson GP, McCaslin DL. A search for evidence of a direct relationship between tinnitus and suicide. J Am Acad Audiol. 2001;12(10):493-6.

36. Pridmore S, Walter G, Friedland P. Tinnitus and suicide: recent cases on the public record give cause for reconsideration. Otolaryngol Head Neck Surg. 2012;147(2):193-5.

Received August 3, 2015 Accepted in revised form September 4, 2017 